\title{
Lecciones de una investigación integrativa sobre sustentabilidad urbana: el proyecto "Risk Habitat Megacity" en Santiago de Chile, 2006-2010
}

Jonatban Barton. Pontificia Universidad Católica de Chile, Santiago, Chile. Jürgen Kopfmüller. Karlsruhe Institute of Technology-Institute for Technology Assessment and Systems Analysis, Karlsruhe, Alemania.

\section{El proceso de investigación como objeto de reflexión}

Existe acuerdo en cuanto a que se necesita de una investigación más integrada para enfrentar la complejidad de los desafíos socioecológicos y socioeconómicos. Una investigación así concebida, especialmente importante desde la perspectiva del desarrollo sustentable, incluye reflexiones sobre justicia, problemas globales y un enfoque dinámico antes que cortoplacista (Newman, 2006). El consenso en torno a este punto ha facilitado arquitecturas de proyectos de investigación cada vez más complejas, más allá de las fronteras nacionales y de torres de marfil científicas. Ejemplo de ello es el séptimo Programa Marco de Investigación de la Unión Europea (Bammer, 2008). Sin embargo, las conclusiones sobre la calidad académica o la productividad de tales iniciativas raramente son consideradas, más allá de publicaciones científicas individuales.

Este trabajo aborda la iniciativa interdisciplinaria germano-chilena Risk Habitat Megacity (RHM), 2006-2010, dirigida al
Gobierno Regional (GORE) de la Región Metropolitana de Santiago (con el que se firmó un acuerdo de colaboración), y coordinada por el Centro Helmholtz de Alemania para Investigación Ambiental (UFZLeipzig). El equipo estuvo compuesto por cuatro centros de investigación de la Asociación Helmholtz, universidades de Santiago (Pontificia Universidad Católica de Chile, Universidad de Chile, Universidad Alberto Hurtado) y de Valparaíso (Pontificia Universidad Católica de Valparaíso) y la Comisión Económica para América Latina y el Caribe de las Naciones Unidas (CEPAL). Lo particularmente interesante aquí no son las conclusiones de la investigación (Krellenberg, Kopfmüeller, Barton \& Heinrichs, 2010), sino el proceso mismo de investigación, que a menudo define la calidad de esta. Entregamos algunas reflexiones sobre este proyecto $\mathrm{y}$ aquellas lecciones aprendidas que son relevantes para otros proyectos. Estas reflexiones buscan clarificar los mecanismos frecuentemente ocultos por los cuales los proyectos de investigación son concebidos, dejando atrás productos que pueden no ser influyentes y que posiblemente no serán evaluados en

E-mail: Jonathan Barton, jbarton@uc.cl|Jürgen Kopfmüller, juergen.kopfmueller@kit.edu 
su totalidad, sino más bien como fragmentos publicables, como se da en el sistema contemporáneo de investigación (Corley, Boardman \& Bozeman, 2006, p. 981).

\section{Antecedentes y objetivos del proyecto RHM}

En particular, la megaurbanización se caracteriza por su creciente complejidad, interdependencias y dinámicas. Es una tendencia global que genera riesgos y oportunidades para alcanzar objetivos de sustentabilidad global, nacional o local. Aunque muchos proyectos científicos previos o en curso se hacen cargo de problemas de desarrollo sustentable urbano, la generación de un adecuado conocimiento orientado a la acción exige que se investigue aún más. Este tipo de investigación puede ser considerado como "de alto riesgo" por patrocinadores e investigadores, por lo difícil de reunir a diferentes instituciones y disciplinas (Brunckhorst, 2005). En lugar de fortalecer la disciplina de planificación para mejorar la investigación transdisciplinaria (Pinson, 2004), los autores — siguiendo a Ramadier (2004) - consideran la planificación y la geografía como "disciplinas puente", que obtienen su fuerza de la conectividad antes que de la exclusividad, dirigiéndolas a la vez hacia temáticas y metodologías interdisciplinarias.

La iniciativa RHM seleccionó como su objeto de investigación la región latinoamericana, porque en ella se encuentran muchas grandes ciudades que ya han alcanzado un punto de inflexión en su desarrollo espacial y demográfico. Por lo tanto, se esperaba que las experiencias de megaciudades latinoamericanas en la lucha contra sus problemas demostraran ser aleccionadoras para otras grandes aglomeraciones urbanas en todo el mundo. Se escogió la Región Metropolitana de Santiago (RMS) porque concentra el 40$50 \%$ del total de la población chilena y del
PIB, tiene problemas representativos del desarrollo urbano y ofrece un excelente marco para la cooperación en la investigación.

El objetivo básico del proyecto era desarrollar y poner a prueba una metodología que permitiese una mejor comprensión del desarrollo megaurbano, considerando objetivos de sustentabilidad, riesgos y estrategias de gobernanza. Con este fin, se desarrolló y aplicó un enfoque de investigación de procesos dirigido tanto a aportar en los debates científicos como a mejorar el capital de conocimientos de los encargados de la toma de decisiones.

\section{El enfoque de investigación de procesos de la iniciativa RHM}

La iniciativa RHM comprendió el trabajo de investigadores asociados en Alemania y Chile con el GORE, para concertar tópicos y metodologías en un enfoque consensuado, el cual estuvo compuesto por cuatro elementos clave de integración. Primero, tres conceptos transversales (CT), como un marco común: "desarrollo sustentable", como la dimensión-objetivo, según el Concepto de Sustentabilidad Integradora de la Asociación Helmholtz (véase Kopfmüller, Brandl \& Jörissen, 2001); “riesgo", focalizado en los problemas que afectan la sustentabilidad; "gobernanza", concentrada en las acciones requeridas. Estos tres conceptos tuvieron varios campos de aplicación $(\mathrm{CdA})$ típicos de megaciudades: calidad del aire, energía, ordenamiento territorial, diferenciación socioespacial, transporte, residuos y agua.

El segundo elemento de integración consistió en una selección y aplicación sistemática de indicadores de sustentabilidad y principios buscados para el año 2030. Una tercera herramienta de integración fue el desarrollo y aplicación común de un enfoque sistemático de escenarios para tratar apropiadamen- 
te con la complejidad y la incertidumbre ligadas al futuro del desarrollo megaurbano. El cuarto elemento de integración consistió en el enfoque inter y transdisciplinario. De acuerdo con estas características de sustentabilidad, se involucraron científicos de diferentes disciplinas (incluyendo las "disciplinas puente"), además de grupos de interés, como los responsables de la toma de decisiones de distintos niveles gubernamentales y representantes de la sociedad civil.

Lo que distingue esta iniciativa de la mayoría de proyectos similares es su enfoque de investigación de procesos, que la hace un ejemplo de investigación "no reduccionista" (Brunckhort, 2005) y permite la coproducción de conocimiento entre investigadores, patrocinadores y usuarios (Roux, Stirzaker, Breen, Lefroy \& Cresswell, 2010).

Los elementos de integración mencionados son una premisa crucial para tratar apropiadamente los problemas del desarrollo urbano sustentable, aunque la pretensión de aplicarlos plenamente implica manejar varios fenómenos, que definimos como "diversidades". La diversidad, como la diversidad cultural o la biodiversidad, es uno de los principios orientadores de la sustentabilidad, y en el contexto de un proyecto como RHM lleva a diversos desafíos.

\section{Diversidades y su relevancia para la investigación de procesos}

Las diversidades que observamos, y que se discuten en las siguientes subsecciones, tuvieron enorme influencia en la investigación de RHM y en la organización interna del proyecto, y están cada vez más presentes en los proyectos internacionales de investigación que pretenden ser integradores en su búsqueda de aprovechar las diferencias (diversidades, en nuestra terminología), establecer límites defendibles y ser legitimados (Bammer, 2008).

\section{Disciplinas y metodologías}

El trabajo interdisciplinario se ve fuertemente respaldado por universidades y patrocinadores. Sin embargo, es un potencial campo minado que puede llevar a la fragmentación $\mathrm{y}$ a una comunicación insuficiente. A menudo los diferentes conocimientos compiten entre sí, por lo que se requieren participantes con experiencia y disposición para trascender sus disciplinas y herramientas disciplinarias. Youngblood (2007) subraya la importancia de ir más allá de los dominios académicos hacia un "pensamiento crítico orientado a los problemas, enfocado en los procesos" (p. 2). Según Pohl (2005), esto debiese ser facilitado por alianzas entre "engaged problem-solvers" (solucionadores-de-problemas comprometidos) y "detached specialists" (especialistas desvinculados). Tales componentes pueden dar valor agregado al trabajo interdisciplinario en comparación con la investigación disciplinaria, pero su operatividad exige un adecuado marco común.

Todo proyecto de investigación requiere un diseño compartido para que los participantes estructuren su trabajo en función de ese marco. Esto es evidente. Sin embargo, para una "buena" investigación, un diseño común debe agrupar diferentes disciplinas, epistemologías, metodologías, "lenguajes" y axiomas. Consecuentemente, el capital social inicial en la fase de diseño del proyecto es decisivo, y de ahí la importancia de los contactos interpersonales o de la motivación intrínseca - la construcción de colaboraciones interpersonales de largo plazo (Sargent \& Waters, 2004) -, en contraposición a los factores puramente instrumentales (habilidades específicas, conocimientos complementarios).

A lo largo del proyecto RHM se insistió en la arquitectura común y en los vínculos entre los CdA y los CT para asegurar que el "pegamento" de la iniciativa persistiera y 
que los grupos de especialistas disciplinarios siguieran el marco integrador, teniendo como plataforma indicadores y escenarios. No es fácil generar los elementos vinculantes de la investigación sobre sustentabilidad cuando están involucradas distintas perspectivas disciplinarias, pues las multi, inter y transdisciplinas implican diferentes desafíos. Sin embargo, la incorporación de "especialistas" e "integradores" hace necesario un entendimiento común del marco para asegurar que el conocimiento reunido sea legible para quienes toman decisiones, para profesionales y otros grupos de interés. La meta es generar un marco flexible capaz de facilitar una mejor comprensión de los sistemas complejos.

\section{Escalas}

Se decidió trabajar en la RMS porque es un sistema en el que los vínculos entre los subsistemas socioeconómico y socioecológico son más evidentes (Brunckhorst, 2005). Existen múltiples escalas mediante las cuales se forman relaciones, dado el contexto de los diferentes CdA. En el caso de la RMS, comprende 52 municipalidades, mientras el Área Metropolitana cuenta con 34. Dada la perspectiva urbana del trabajo fue difícil mantener un foco regional, a pesar de la importancia de la región en términos de - por ejemplo- la gestión de recursos hídricos y las proyecciones para 2030. En el caso de la energía, la región es parte de un sistema nacional de transmisión, por lo que el análisis debió centrarse principalmente en esta escala.

Diferentes CdA (transporte y diferenciación socioespacial) fueron tratados en un rango intermedio, al considerarse en la investigación una nueva Área Metropolitana, producto de la expansión periurbana hacia 2030, con 38 y 39 municipalidades. En el caso del ordenamiento territorial, solo se analizaron 33 municipalidades, debido a la limitada información satelital para una comuna en particular. Una mayor adaptación de escalas ocurrió al fusionar las municipalidades del Área Metropolitana en grupos similares, para analizar la diferenciación socioespacial. Esto revela las dificultades de generar una escala de análisis consensuada y de producir resultados comparables e integradores.

\section{Instituciones y culturas}

La participación de diversas instituciones, incluyendo universidades, una empresa privada (Ingeniería Alemana), instituciones políticas y de gobierno, generó diversas exigencias de coordinación. Entre ellas, la consideración de los costos de oportunidad en la participación, las restricciones de tiempo para estas contribuciones y las culturas financieras y de trabajo de cada una de las instituciones involucradas. Así, en la estructura del proyecto debió considerarse el tiempo suficiente para gestar el desarrollo y la ejecución del mismo, estructuras de coordinación claras y vías para la legitimación de decisiones y de grupos representativos.

La arquitectura común de RHM definió la organización de grupos temáticos y sus interrelaciones, aunque las maneras en que ellos funcionaron dependieron de la formulación de la institucionalidad del sistema, esto es, las normas, prácticas y reglas compartidas. Ellas fueron establecidas mediante cuatro “ajustes” para asegurar el liderazgo, la responsabilidad y la coordinación: rendición de cuentas (accountability) institucional (un Comité de Dirección Científica), rendición de cuentas del proyecto (un Consejo Científico Asesor), coordinación del proyecto (una organización líder con un vocero alemán y uno chileno en cada tema) y la interfaz proyecto-usuario (difusión de información, sitio web, conferencias y talleres). El uso de acuerdos firmados fue el punto de 
partida para garantizar el compromiso y la transparencia.

Estos ajustes formaron la arquitectura del proyecto y fueron decisivos para asegurar su cohesión y seriedad ante patrocinadores y usuarios. Sin embargo, los participantes del proyecto, tanto personas naturales como representantes de instituciones, trabajaron para diferentes organizaciones y tuvieron distintos compromisos con la iniciativa, dependiendo de la asignación de tiempo y del financiamiento. Esto, combinado con la rotación de participantes durante cinco años, generó una amplia gama de responsabilidades. Un problema mayor fue el lenguaje, con el idioma inglés como la lengua del proyecto y el español como la de usuarios y grupos de interés. Dadas las implicancias que el uso de diferentes lenguas tiene sobre el tiempo de un proyecto y sobre la comunicación, esto no debiese ser subestimado como un factor en su diseño.

\section{Grupos de interés}

Otro elemento crucial para proyectos que tratan con el desarrollo sustentable y apuntan a cambios prácticos es la participación de grupos de interés relevantes, fundamentalmente instituciones de gobierno, agrupaciones de la sociedad civil y empresas. El desafío fue ubicar e invitar a los actores importantes, motivar la participación en las actividades del proyecto, diseñar alternativas de participación adecuadas y manejar los resultados potencialmente controversiales. El GORE fue una importante contraparte política desde el inicio. Aunque no tiene la autoridad ni el presupuesto de las secretarías regionales ministeriales para cada sector del gobierno nacional, debe asegurar la coordinación y desarrollar las prioridades de planificación territorial. Por lo tanto, se lo consideró como la institución más importante para sacar adelante el trabajo. No obstante, dos debilidades institucionales del GORE complicaron esta colaboración: el carácter transitorio de los liderazgos institucionales (rotación frecuente) y la división del poder entre el intendente, la administración regional y el Consejo Regional.

Los grupos de interés participaron en diferentes equipos de trabajo en distintas fases del proyecto, y su rol fue definido por las restricciones de recursos y tiempo de los participantes. En estas condiciones, tomaron parte en la selección de indicadores, en la determinación de valores-objetivo, en el diseño de escenarios, en la discusión de medidas apropiadas para tratar con los problemas previstos para 2030 y en la conferencia final que marcó el fin del proyecto. Se prepararon diferentes actividades con los grupos de interés en función de los grupos de trabajo, aunque es difícil organizar la participación continua de instituciones gubernamentales y no gubernamentales. Ello exige un uso intensivo de recursos, y pasar de discusiones a resultados consensuados involucra un trabajo considerable. Sin embargo, ello es vital para establecer diálogos y compromisos y asegurar la legitimidad del proceso y de las propuestas generadas.

\section{Expectativas y resultados}

Dado que el proyecto se proponía apoyar la toma de decisiones locales, una evaluación en este sentido es necesaria, a la vez que compleja. No está claro el legado de este proyecto, pero es importante establecer cómo se mide el impacto en la toma de decisiones y sobre qué marco temporal, y qué calidad tiene ese impacto (De Jong, Van Arensbergen, Daemen, Van der Meulen \& Van den Besselaar, 2011). El material generado y la comunicación con la División Regional de Planificación, así como la toma de conciencia entre algunos consejeros regionales, sugiere que hay un efecto de ósmosis por el cual los re- 
sultados de la investigación están disponibles y pueden influir sobre futuros instrumentos. Tal fue el caso del proyecto OTAS 1997 2005 (Ordenamiento Territorial Ambientalmente Sustentable). El período de cristalización entre la producción de resultados y su incorporación suele ir más allá del cierre del proyecto. Tremblay, Zohar, Bravo, Potsepp y Barker (2010) señalan que es útil centrarse en diferentes tipos de impactos a intervalos cortos, pues es beneficioso un enfoque gradual en actividades continuas antes que en los impactos finales.

Aunque el informe final del proyecto fue entregado en la conferencia de cierre de la iniciativa en octubre de 2010, dos libros que sintetizan los resultados fueron publicados recién a fines de 2011 (Barton \& Kopfmüller, 2011; Heinrichs, Krellenberg, Hansjürgens \& Martínez, 2011), complementados por un video sobre el caso de Santiago, un atlas y un panorama comparativo regional del desempeño metropolitano de sustentabilidad.

El planteamiento es que los verdaderos impactos del proyecto, y el cumplimiento de diversas expectativas, posiblemente se establezcan de manera posterior a la conclusión formal de la iniciativa misma, y no en el momento preciso del fin de su financiamiento. Este fenómeno, común a muchos proyectos de este tipo, debe ser considerado por quienes son responsables de evaluarlos. Otro factor de la evaluación de impactos es la claridad respecto de las recomendaciones de políticas públicas. Al proponer recomendaciones específicas, sin importar cuán moderadas o radicales sean, debiera ser posible rastrearlas mediante iniciativas futuras. Sin claridad en este punto, será difícil identificar los impactos del proyecto. Además, sin las apropiadas evaluaciones ex post, los patrocinadores sabrán poco acerca de la eficiencia de su contribución. Por lo tanto, la tendencia es buscar mecanismos de evaluación si- milares a los de la investigación disciplinaria, basados en publicaciones, lo que genera diferentes incentivos para los investigadores (Feller, 2006; Sherren, Klovdahl, Robin, Butler \& Dovers, 2009; Van Rijnsoever \& Hessels, 2011). Analizar impactos también involucra un examen de las expectativas del proyecto, las que son diversas, como también lo son los horizontes temporales (por ejemplo, los horizontes de largo plazo de los estudiantes de doctorado frente a las necesidades en el corto plazo de las contrapartes técnicas en el gobierno), por lo que deben ser discutidas abiertamente.

\section{Lecciones aprendidas}

Hay muchas lecciones que aprender del proyecto RHM y de otras investigaciones de procesos orientados a la sustentabilidad. Sin embargo, existe relativamente poca reflexión ex post sobre estas experiencias una vez que el financiamiento termina y se crean nuevos proyectos. Este artículo busca llenar esta brecha de conocimiento y generar la necesidad de concentrarse en los "dominios epistémicos y organizacionales" de la investigación (Corley et al., 2006). Reflexiones similares en otros proyectos basados en Santiago, como OTAS y DIMSUD (20012004; véase Keiner, Zegras, Schmid \& Salmerón, 2004), debieron haber sido útiles en este sentido. Mediante la reflexión, las fortalezas y debilidades de la investigación interdisciplinaria colaborativa pueden ser ventiladas y los procesos pueden ser mejorados, abriendo nuevas líneas de indagación (Youngblood, 2007).

La cultura importa. Las culturas son la base de la organización social, y el viraje desde redes nacionales a internacionales expone esta diversidad cultural en las redes científicas y en el modo en que afecta los procesos de investigación. Las culturas nacionales y locales derivan de identidades, así como las cultu- 
ras de trabajo en diferentes instituciones lo hacen para diferentes personas en distintos puntos de sus carreras, con múltiples intereses y demandas. El trabajo, la organización y la comunicación están también fuertemente influenciados por el lenguaje y la cultura. Estos son temas que deberían hacerse explícitos desde el comienzo de los proyectos en referencia, puesto que requieren de tiempo e inversión adicionales.

La confianza importa. Aunque los objetivos de investigación y el marco común fueron producto de una colaboración inicial entre investigadores alemanes para postular a financiamiento, es valioso reflexionar sobre la importancia de la construcción de capital social. La confianza y honestidad entre los coinvestigadores requiere tiempo, comprensión y tolerancia. La investigación no remite solo a un "hacer", sino también al "entendimiento compartido" por los participantes (acerca de objetivos, obligaciones y responsabilidades), sin el cual los avances bien pueden verse coartados. Pohl (2011, p. 625) define esto como una "comunidad de prácticas".

Las expectativas importan. Todos los proyectos generan expectativas en patrocinadores, coordinadores, usuarios, asesores, y en los propios participantes. Sin embargo, ellas son heterogéneas, difíciles de manejar y cambiantes en el tiempo. Debe haber claridad sobre las expectativas de todos los participantes, y deben ser compartidas y comprendidas para reducir la frustración por su incumplimiento.

Los impactos importan. Para que los resultados de la investigación tengan impacto una vez que el financiamiento termina y han salido a la luz las publicaciones respectivas, se requiere que los usuarios comprendan el material y deseen incorporarlo en sus actividades. Con un proyecto como RHM, enfocado en políticas públicas, la necesidad de encontrar "paladines" (usuarios y beneficiarios del conocimiento, en palabras de Meagher, Lyall \& Nutley, 2008) fue vital, dado que los grupos de interés involucrados son clave para legitimar los resultados. La necesidad de convertir productos parciales en documentos que los participantes pudiesen usar sugiere que el proceso de investigación debiera concentrarse en generar una investigación de corto plazo con objetivos estratégicos de largo plazo. Otro impacto que debe tomarse en cuenta es la capacidad de construir capacidades. Un ejemplo de ello es la experiencia del proyecto ClimateAdaptationSantiago, financiado por el Ministerio Federal de Ambiente, de Alemania, entre 2010 y 2013, el que ha permitido que muchos investigadores de RHM continúen colaborando e interactuando con el sector público a partir del proyecto original.

El tiempo importa. No debería ser una sorpresa que la palabra "tiempo" reaparezca frecuentemente en estas reflexiones, pues es una variable a menudo considerada solo en cuanto a la temporalidad del financiamiento del proyecto y en fechas clave, como las conferencias anuales de actualización o para los resultados finales del proyecto RHM. No obstante, cada uno de los participantes experimenta diferentes presiones y preocupaciones relativas a la temporalidad del proyecto. Así, debe elaborarse tempranamente un programa que considere los diferentes tiempos de los investigadores, de manera de cumplir con los requisitos de transparencia y planificación.

Un marco común importa. En tanto las conexiones entre grupos de interés son clave para la duración y la relevancia de los hallazgos de la investigación más allá del fin del proyecto, también fue evidente la importancia del diseño de investigación y el tiempo disponible para este proceso. El financiamiento inicial que permitió al con- 
sorcio germano-chileno elaborar este marco común fue decisivo, y deben promoverse enfoques similares de patrocinio. Puede haber incertidumbres acerca del modo en que opera el espacio entre las alternativas de un marco común comparado con metodologías diversas y separadas. No obstante, debe coordinarse una metodología central, como la de escenarios y herramientas satélite. Los problemas acerca de la escala también necesitan ser discutidos en este marco para asegurar una comprensión común y una integración efectiva.

Es importante reflexionar sobre el proceso de investigación. Todas las diversidades encontradas y las lecciones aprendidas deben ser consideradas en la planificación y gestión de la investigación. Ello incrementa la complejidad, aunque la investigación de procesos de sustentabilidad busca reconocer tal complejidad y responder a ella. Quizás no sea posible tratar todas las diversidades de igual manera, pero deben estar presentes en el diseño de investigación. Por supuesto que los resultados son centrales en cualquier iniciativa, pero los impactos de tales resultados, los efectos secundarios no deseados de la colaboración y la construcción de capacidades, o las consecuencias de largo o corto plazo, no deben ser pasados por alto. Existe una gran diversidad de temas, y diversidades al interior de cada tema, que deben ser puestas a la luz en los grandes proyectos de investigación. Sin una adecuada reflexión y comunicación en relación con los procesos de investigación y las diversidades que deben manejar proyectos como RHM, es poco probable que futuras colaboraciones de investigación puedan beneficiarse de sus experiencias, marcos y metodologías.

\section{Referencias}

Bammer, G. (2008). Enhancing research collaborations. Three key management challenges. Research Policy, 37(5), 875-887. doi:10.1016/j.respol.2008.03.004

Barton, J. \& Kopfmüller, J. (2011). Diversity in integrative urban sustainability research. Lessons from the 'Risk Habitat Megacity' project on Santiago de Chile, 2006-10. Working Paper.

Barton, J. \& Kopfmüller, J. (Eds.) (En prensa). Santiago 2030. ¿Hacia la sustentabilidad? Santiago: EURE Libros.

Brunckhorst, D. (2005). Integration research for shaping sustainable regional landscapes. Journal of Research Practice, 1(2), 1-24. Disponible en http://jrp.icaap.org/index.php/jrp/article/ view/16/28

Corley, E. A., Boardman, P. C. \& Bozeman, B. (2006). Design and the management of multi-institutional research collaborations: theoretical implications from two case studies. Research Policy, 35(7), 975-993. doi:10.1016/j.respol.2006.05.003

De Jong, S. P. L., Van Arensbergen, P., Daemen, F., Van der Meulen, B. \& Van den Besselaar, P. (2011). Evaluation of research in context. An approach and two cases. Research Evaluation, 20(1), 61-72. doi: http://dx.doi.org/10.3152/095820211X12941371876346

Feller, I. (2006). Multiple actors, multiple settings, multiple criteria. Issues in assessing interdisciplinary research. Research Evaluation, 15(1), 5-15. doi: http://dx.doi .org/10.3152/147154406781776020

Heinrichs, D., Krellenberg, K., Hansjürgens, B. \& Martínez, F. (Eds.) (2011). Risk Habitat Megacity. The case of Santiago de Chile. Dordrecht: Springer.

Keiner, M., Zegras, C., Schmid, W. A. \& Salmerón, D. (Eds.) (2004). From understanding to action. Sustainable urban development in medium-sized cities in Africa and Latin America. Dordrecht: Springer. 
Kopfmüller, J. Brandl, V. \& Jörissen, J. (2001). Nachhaltige Entwicklung Integrativ Betrachtet: Konstitutive Elemente, Regeln, Indikatoren. Berlin: ITAS.

Krellenberg, K.; Kopfmüeller, J., Barton, J. \& Heinrichs, D. (2010). Introduction. En VV.AA. How sustainable is Santiago de Chile: synthesis report. Leipzig: UFZ Report.

Meagher, L., Lyall, C. \& Nutley, S. (2008). Flows of knowledge, expertise and influence: a method for assessing policy and practice impacts from social science research. Research Evaluation, 17(3), 163-173. doi: http://dx.doi.org/10.3152/095820208X331720

Newman, L. (2006). Change, uncertainty, and futures of sustainable development. Futures, 38, $633-$ 637.

Pinson, D. (2004). Urban planning: an 'undisciplined' discipline? Futures, 36, 503-513.

Pohl, C. (2005). Transdiscplinary collaboration in environmental research. Futures, 37, 1159-1178.

Pohl, C. (2011). What is progress in transdiscplinary research. Futures, 43, 618-626.

Ramadier, T. (2004). Transdiscplinarity and its challenges: the case of urban studies. Futures, 36, 423439.

Roux, D. J., Stirzaker, R. J., Breen, C. M., Lefroy, E. C. \& Cresswell, H. P. (2010). Framework for participative reflection on the accomplishment of transdisciplinary research programs. Environmental Science and Policy, 13(8), 733-741. doi: 10.1016/j.envsci.2010.08.002

Sargent, L. \& Waters, L. (2004). Careers and academic research collaborations: an inductive process Framework for understanding successful collaborations. Journal of Vocational Behaviour, 64(2), 308-319. doi:10.1016/j.jvb.2002.11.001

Sherren, K., Klovdahl, A. S., Robin, L., Butler, L. \& Dovers, R. (2009). Collaborative research on sustainability: myths and conundrums of interdisciplinary departments. Journal of Research Practice, 5(1), 1-29. Disponible en http://www.eric.ed.gov/PDFS/EJ838432.pdf

Tremblay, G., Zohar, S., Bravo, J., Potsepp, P. \& Barker, M. (2010). The Canada Foundation for Innovation's outcome measurement study: A pioneering approach to research evaluation. Research Evaluation, 19(5), 333-345. doi: 10.3152/095820210X12809191250960

Van Rijnsoever, F. \& Hessels, L. (2011). Factors associated with disciplinary and interdisciplinary research collaboration. Research Policy, 40(3), 463-472. doi:10.1016/j.respol.2010.11.001

Youngblood, D. (2007). Interdisciplinary studies and the bridging disciplines. A matter of process. Journal of Research Practice, 3(2), 1-8. Disponible en http://www.eric.ed.gov/PDFS /EJ800366.pdf 